\title{
Transdifferentiation-inducing HCCR-1 oncogene
}

\author{
Seon-Ah Ha+1, Hyun K Kim', JinAh Yoo', SangHee Kim¹, Seung M Shin'1, Youn S Lee², Soo Y Hur³, Yong W Kim³, \\ Tae E Kim³, Yeun J Chung ${ }^{4}$, Shin S Jeunn, Dong W Kim6, Yong G Park7, Jin Kim8, Soon Y Shin'9, Young H Lee ${ }^{9}$ and \\ Jin W Kim*1,3
}

\begin{abstract}
Background: Cell transdifferentiation is characterized by loss of some phenotypes along with acquisition of new phenotypes in differentiated cells. The differentiated state of a given cell is not irreversible. It depends on the up- and downregulation exerted by specific molecules.

Results: We report here that HCCR-1, previously shown to play an oncogenic role in human cancers, induces epithelialto-mesenchymal transition (EMT) and mesenchymal-to-epithelial transition (MET) in human and mouse, respectively. The stem cell factor receptor CD117/C-Kit was induced in this transdifferentiated (EMT) sarcoma tissues. This MET occurring in HCCR-1 transfected cells is reminiscent of the transdifferentiation process during nephrogenesis. Indeed, expression of HCCR-1 was observed during the embryonic development of the kidney. This suggests that HCCR-1 might be involved in the transdifferentiation process of cancer stem cell.
\end{abstract}

Conclusions: Therefore, we propose that HCCR-1 may be a regulatory factor that stimulates morphogenesis of epithelia or mesenchyme during neoplastic transformation.

\section{Background}

The concept that genetic events cooperate to achieve malignant transformation was proposed over a decade ago. Primary rodent cells are efficiently converted into tumorigenic cells by the co-expression of cooperating oncogenes. However, similar experiments with human cells have consistently failed [1]. In 1999, after more than 15 years of trying, researchers have managed to convert normal human cells into tumor cells by delivering telomerase catalytic subunit in combination with two oncogenes [2]. Although malignant transformation of human cells by a single oncogene may not occur or may require specialized factors, we demonstrated that HCCR-1, associated with various types of human cancers, alone induced tumorigenic conversion of mouse cells [3].

We have identified a novel oncogene, human cervical cancer oncogene (HCCR), that was classified into 2 types: HCCR-1 (GenBank accession number AF 195651) and HCCR-2 (GenBank accession number AF 315598) [3]. The HCCR-1 and HCCR-2 overexpressed cells were tum-

\footnotetext{
* Correspondence: jinwoo@catholic.ac.kr

1 Molecular Genetic Laboratory, Catholic Medical Research Institute, The

Catholic University of Korea, Seoul, Korea

+ Contributed equally

Full list of author information is available at the end of the article
}

origenic in nude mice and $H C C R$ transgenic mice developed breast cancers and metastasis [3,4]. Also, HCCR-1 was overexpressed in various types of human malignancies and was found to regulate the p53 tumor-suppressor gene negatively [3-6]. However, it is unknown how HCCR-1 contributes to the cellular and biochemical mechanisms of human tumorigenesis.

Cell transdifferentiation is characterized by loss of some phenotypesalong with acquisition of new phenotypes in differentiated cells. Differentiated cells are endowed with the capacity of transforming into cells of a different type having other functions [7]. Gene expression in differentiated cells has long been considered an irreversible phenomenon that is established at the time of replication. Given that, although repressed, the same genetic framework is present in all cell types, a change in gene expression among differentiated cells was predictable in particular conditions.In fact, the differentiated state of a given cell is not irreversible.It depends on the up- and downregulation exerted by specificmolecules [8].

Recent research suggests that tumor formation may result from the development of cancer stem cells by the deregulation of normal self-renewal pathways of tissue stem cells. Numerous signalling pathways have been implicated in this process including Notch, Wnt, LIF (leu- 
kemia inhibitory factor), PTEN (phosphatase and tensin homologue deleted from chromosome 10), SHH (sonic hedgehog) and BMI1 [9-12]. The discovery of cancer stem cells in AML, breast cancer and some CNS tumors offers a new approach to understanding the biology of these conditions. Further study into these and other mechanisms controlling self-renewal pathways is needed to understand not only what drives tumor formation from cancer stem cells but also what mechanisms could be used to 'switch off' tumor formation [13].

We undertook this study with the following aims: 1) to assess whether HCCR-1 overexpression converts normal cells to malignant transformed cells; 2) to determine whether HCCR-1 is involved in transdifferentiation process and embryonic kidney development; 3) to examine the molecular alterations occurring in HCCR-1 induced tumorigenesis.

\section{Results}

HCCR-1 is involved in tumorigenesis and transdifferentiation

We investigated whether $H C C R-1$ alone can induce malignant transformation of HEK-293 cells. Transfection of HCCR-1 expression vector into HEK-293 cells yielded a transformation efficiency (i.e., the number of foci per $\mu \mathrm{g}$ of DNA transfected) of 0.9 (Table 1). The transformation efficiency of the wild-type ras gene used as a positive control was 1.2 (95\% CI, 16.9-19.1) (Table 1). These data suggest that $H C C R-1$ has almost as potent oncogenic activity as wild-type ras in the HEK-293 assay system. Comparable transfection with the control vector alone (nontransformed 293 cells) yielded no transformation (Table 1). In total, 20 of the transformed foci were isolated from the HCCR-1 transfected 293 cells and were grown in semisolid soft-agar medium to measure the colony formation efficiency. HCCR-1-transfected 293 cells formed colonies with an efficiency of $13 \%$.

HEK-293 is a short spindle shaped cell having a bipolar cell process (Figure 1A). Cultured 293 cells have similar cytological features to cells transfected with vector alone (Figure 1B). However, HCCR-1-transfected human 293 cells showed increase in cell size compared to wild-type human 293 cells (Figure 1C). The cell processes were blunted in HCCR-1-transfected human 293 cells. Northern blot showed that about 2.0-kilobase-pair mRNA transcript was over-expressed in all five HCCR-1-transfected human 293 cells as compared with wild-type 293 cells (Figure 1D). Nude mice injected with HCCR-1-transfected human 293 cells showed palpable tumors in four weeks. But cells transfected with vector alone did not induce tumor formation in nude mice. Sections of the nude mice tumor nodules bearing HCCR-1-transfected human 293 cells revealed epithelial cell carcinomas (Figure 1E). Interestingly, however, the cells showed coexpression of epithelial markers, such as cytokeratin 8 (Figure 1F) and cytokeratin 19 (Figure 1G), and of the mesenchymal marker, vimentin that is normally expressed by fibroblasts (Figure $1 \mathrm{H}$ ). These results suggest that transdifferentiation (EMT) occurred in HCCR-1 stably transfected 293 cells derived from nude mouse tumors.

In order to confirm the above data, we performed the immunofluorescence microscopy experiment in HEK293 and HEK-293 stable clone for HCCR-1 (Additional file 1 Figure S1 A, B). The result showed that the parental HEK-293 cells express the vimentin but in a lower level than HCCR-1 stable HEK-293 clone. This suggests that the vimentin level is slightly increased after HCCR-1 transfection. We then analyzed other epithelial markers in HEK-293, HEK-293 clones stably transfected with HCCR-1, and HEK-293 cells transfected with the empty vector. $E$-cadherin, $\alpha$-catenin, and $\beta$-catenin are essential for the maintenance of epithelial structures. To determine whether the over-expression of HCCR-1 alters the expression profiles of these molecules, we performed the western blotting analyses as follows (Additional file 2 Figure $\mathrm{S} 1 \mathrm{C}$ ). The result demonstrates that all of these epithelial markers (E-cadherin, $\alpha$-catenin, and $\beta$-catenin) are down-regulated only in $H C C R-1$ stable cell lines. Therefore, this data support that HCCR-1 induces EMT in transformed HEK-293 cells.

Table 1: Transforming activity of HCCR-1 in HEK-293 cells

\begin{tabular}{|c|c|c|c|}
\hline Transfected DNA source & 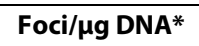 & Transformation efficiency** & 95\% confidence intervals \\
\hline HCCR-1 & $13 / 15$ & 0.9 & $11.2-13.8$ \\
\hline Wild-type ras & $18 / 15$ & 1.2 & $16.0-19.1$ \\
\hline Vector alone & $0 / 15$ & 0 & - \\
\hline
\end{tabular}



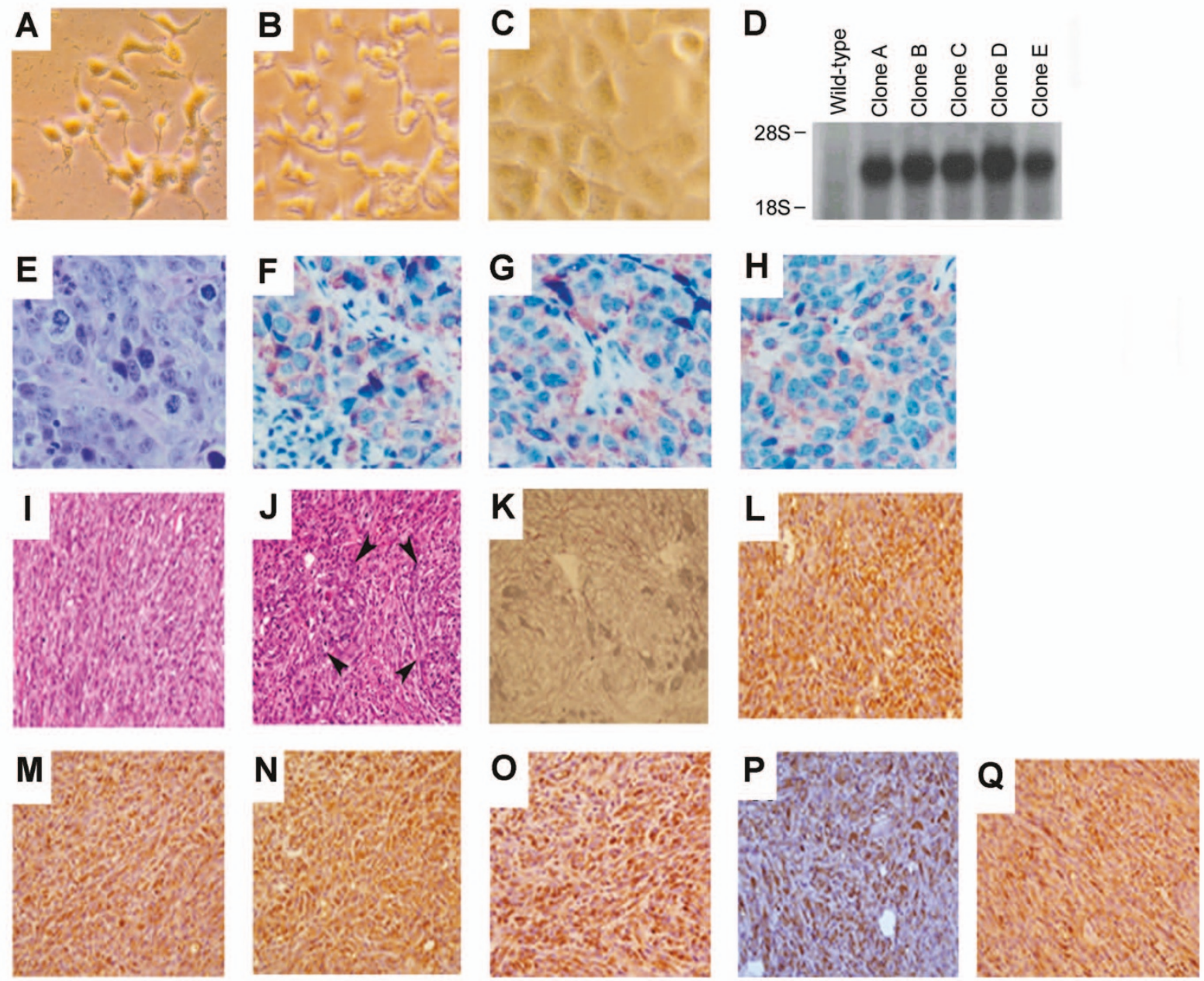

Figure 1 HCCR-1 is ivolved in tumorigenic conversion and transdifferentiation. Phase-contrast features of wild-type 293 cells (A), cells transfected vector alone (B) and HCCR-1-transfected human 293 cells (C). D. HCCR-1 mRNA expressions in HCCR-1-transfected human 293 cells (designated as clone A, B, C, D, and E, consecutively), and wild-type human 293 cells. E. Hematoxylin-eosin (H-E) staining of tumor nodules taken from the nude mice xenograft. The tumor cells show similar histological features with HCCR-1-transfected allografts. Tumor cells are arranged in nests separated by fine fibrovascular septae and have polygonal shape with oval nuclei, coarse chromatin and moderate amount of cytoplasm. Numerous mitotic figures are found. Immunohistochemical stainings of HCCR-1-transfected human 293 cells xenograft. Tumor cells show positive cytoplasmic stainings for cytokeratin $8(\mathbf{F})$, cytokeratin $19(\mathbf{G})$, and vimentin $(\mathbf{H})$, respectively. Magnifications, $\mathbf{E}) \times 300 ; \mathbf{F}-\mathbf{H}) \times 250$. Tumorigenic conversion and transdifferentiation of $\mathrm{NIH} / 3 \mathrm{~T} 3$ cells after transfection with HCCR-1. I and J. H-E staining of tumor nodules taken from nude mice after 3 weeks injection with HCCR-1-transfected NIH/3T3 cells. [I, tumor sections show malignant spindle cell sarcoma; J, tumor sections show poorly differentiated sarcoma with focal epithelial differentiation (arrows)]. Magnification $\times$ 400. K. Reticulin stain was performed to detect reticulin fibers. Sections from the same tumor nodule were immunohistochemically stained with mouse monoclonal antibodies (DAKO) specific for epithelial membrane antigen (L), cytokeratin 7 (M), cytokeratin $8 \mathbf{( N )}$, cytokeratin $19(\mathbf{O})$, cytokeratin 20 (P), and vimentin (Q). DAB was used as the chromogen. After immunostaining, sections were counterstained with hematoxylin. Magnification $\times 200$.

The oncogenic transformation of NIH/3T3 cells would typically be expected to develop sarcomas because NIH/ 3T3 cells are of mesenchymal origin [14]. However, the tumors in nude mice derived from HCCR-1 stably transfected NIH/3T3 cells had both sarcomatous (Figure 1I) and epithelial features (Figure 1J). Histologically, the tumors showed poorly differentiated sarcoma with epithelial differentiation (carcinosarcoma) (Figure 1J). They were composed mostly of spindle cells forming long and short fasciles. In focal areas these cells were vaguely aggregated to form epithelial cell nest-like structures (arrows). Reticulin fibers enveloped individual spindle cells in the sarcomatous areas, but enveloped vague epithelial cell nests in the more carcinomatous areas (Figure $1 \mathrm{~K})$. For a morphologic comparison between HCCR-1derived tumor cells and epithelial cells, we determined 
whether HCCR-1-derived tumor cells expressed epithelial cell markers, such as the epithelial membrane antigens (Figure 1L), cytokeratin 7 (Figure $1 \mathrm{M}$ ), cytokeratin 8 (Figure $1 \mathrm{~N}$ ), cytokeratin 19 (Figure 1O), cytokeratin 20 (Figure 1P), and the mesenchymal marker vimentin (Figure 1Q). HCCR-1-derived tumor cells were positive for both epithelial and mesenchymal markers (Figures 1L1Q). These results suggest that transdifferentiation (MET) occurred in HCCR-1 stably transfected NIH/3T3 cells derived from nude mice tumors. HCCR-1 might play multiple developmental roles by mediating a signal originating from the mesenchyme and received by epithelia. Mesenchymal signals are known to govern differentiation and morphogenesis of many epithelia, but the molecular nature of the signals is poorly understood. This expression pattern indicates that this mesenchymal factor can transmit morphogenetic signals in epithelia development and suggests a molecular mechanisim for mesenchymal epithelial interactions. This study indicates that the HCCR-1 oncogene may be a mesenchyme-derived cytokine that stimulates the morphogenesis of epithelia and mediates interactions between the mesenchyme and epithelia during neoplasia.

\section{Induction of c-kit proto-oncogene product by HCCR-1}

CD117 (c-Kit) is a type III receptor tyrosine kinase operating in cell signal transduction in several cell types. Normally c-Kit is activated (phosphorylated) by binding of its ligand, the stem cell factor (SCF) [15]. This leads to a phosphorylation cascade ultimately activating various transcription factors in different cell types. Such activation regulates apoptosis, cell differentiation, proliferation, chemotaxis, and cell adhesion. Ligand independent activation of c-Kit (dysregulated kit function) has been found to be an important component of oncogenesis in a large number of neoplastic disorders such as systemic mastocytosis, germ cell tumors, acute myelogenous leukemia (AML) with the disruption of the core binding factor, amongst others [16]. C-Kit positivity has been variably reported in sarcomas [16]. Among mesenchymal tumors, c-kit seems to be specific for the gastrointestinal stromal tumors (GISTs), which consistently express this protein. These tumors uniformly express CD117, the $c$-Kit protooncogene product [15]. Signal transductions from tyrosine kinase receptors have key roles in the regulation of cellular proliferation and differentiation [17] and binding of SCF to c-Kit activates multiple signal transduction pathways, including phosphatidylinositol 3-kinase (PI3K)/Akt [18]. Because HCCR-1 induced carcinosarcoma and HCCR-1 is regulated by the PI3K/Akt signaling pathway [19], we investigated whether HCCR-1-induced nude mice-derived carcinosarcoma induces the expression of CD117, as does GIST. HCCR-1-derived tumor cells showed a positive staining for CD117 (Figure 2A).
A

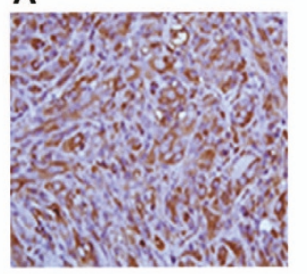

B

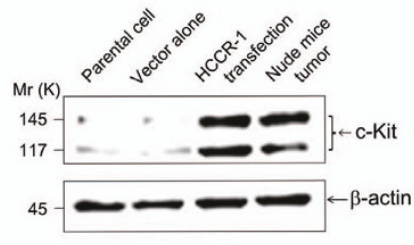

Figure 2 HCCR-1 induced the c-kit proto-oncogene product

HCCR-1 induced the $c$-kit proto-oncogene product. A. Sections were stained for CD117. B. Western blot analysis to determine the expression of c-kit proto-oncogene product. HCCR-1 gene in embryonic kidney development.

Western blot analysis also showed that NIH/3T3 cells stably transfected with $H C C R-1$ and nude mice-derived tumors injected with NIH/3T3 cells transfected with $H C C R-1$ both overexpressed the c-kit protein compared with NIH/3T3 parental cells or cells transfected with vector alone (Figure 2B). Our study shows that HCCR-1derived tumor cells express CD117, suggesting that $H C C R-1$ is related to the c-kit signaling pathway.

\section{Embryonic kidney development}

Co-expression of the human Met receptor, its ligand, and hepatocyte growth factor/scatter factor (HGF/SF), in $\mathrm{NIH} / 3 \mathrm{~T} 3$ fibroblasts causes cells to become tumorigenic in nude mice. The resultant tumors display lumen-like morphology, contain carcinoma-like focal areas with intercellular junctions resembling desmosomes, and coexpress epithelial (cytokeratin) and mesenchymal (vimentin) cytoskeletal markers. The apparent MET of the tumor cells mimics the conversion that occurs during embryonic kidney development, suggesting that Met$\mathrm{HGF} / \mathrm{SF}$ signaling plays a role in this process as well as in tumors that express both epithelial and mesenchymal markers [20]. Because acquisition of epithelial properties by the fibroblast-derived cells mimics the MET of cells during the organogenesis of the kidney [20], we investigated whether HCCR-1 is expressed in the developing kidney. Immunoblot analysis demonstrated that as probed by rabbit polyclonal anti-HCCR-1 serum, HCCR1 began to be overexpressed at fetal 18-day, remaining high up to postnatal 14-day, and decreased to a very low level in adult rat kidney (Additional file 2 Figure S2 A). Sections of 20-day-old fetal rat kidney revealed that HCCR-1 antibody stained throughout the collecting ducts only (Additional file 1 Figure S2 B, medulla on the left side), which are derived from the ureteric bud [21]. The developing nephrons in the cortex were not stained (Additional file 1 Figure S2 B, nephrogenic zone on the right side). But the basolateral plasma membranes of the 
developing collecting duct, which are derived from the ureteric bud, were especially reactive to HCCR-1 antibody (Additional file 1 Figure S2 C). Because nephrogenesis is stimulated by a distinct ureteric signal, diffusionlimited basolateral molecules [22], which trigger MET, we propose that the $H C C R-1$ product may be a mesenchyme-derived regulatory factor [23] that stimulates morphogenesis of epithelia in the developmental process and mediates interactions between mesenchyme and epithelia during neoplastic transformation.

\section{Molecular genetic alterations in the HCCR-1-induced tumorigenesis}

In order to study whether there was an alteration in the growth properties of HCCR-1-transfected cells, we examined cell cycle profiles. The percentage of wild-type NIH/ 3T3 cells and HCCR-1-transfected cells in the S-phase was $20.6 \%$ and $31.5 \%$, respectively (Figure $3 \mathrm{~A}$, mid-log phase). These results suggest that there was a significant shift of the cell population out of the $\mathrm{G}_{0} / \mathrm{G}_{1}$-phase into the S-phase in HCCR-1-transfected cells. To assess the serum-dependent cell cycle progression, cells were cultured in $0.5 \%$ bovine calf serum (BCS) for 36 hours. After incubation, cells were released with $20 \%$ serum and harvested at the indicated times. In wild-type cells (measured at 0 hour), few cells remained in the S-phase (8\%). In contrast, a considerable number of HCCR-1-transfected cells were still in the S-phase (21.8\%), suggesting that constitutive overexpression of $H C C R-1$ allowed for a relative amount of resistance to serum deprivationinduced $G_{0} / G_{1}$ arrest. Following the release of cells from the growth arrest caused by serum-deprivation, there were consistent increases of over $10 \%$ in the S-phase populations of HCCR-1-transfected cells as compared to wild-type cells at measured time intervals (24 hour and 48 hour, respectively). Therefore, overexpression of HCCR-1 could deregulate cell growth by shortening the $\mathrm{G}$ ${ }_{0} / \mathrm{G}_{1}$-phase and increasing the $\mathrm{S}$-phase population of cells. It has been implicated that egr-1 functions as a tumor-suppressor [24]. To assess whether HCCR-1induced tumor formation is associated with the loss of egr-1 expression, we tested the time course of egr-1 expression. When quiescent cells were stimulated with $20 \%$ serum, a marked down-regulation of egr-1 was observed in HCCR-1-transfected cells compared with wild-type NIH/3T3 cells (Figure 3B). In contrast, upregulation of GAPDH mRNA level in HCCR-1-transfected cells was clearly seen, while no significant difference was observed in the level of c-fos (Figure 3B). This result suggests that down-regulation of tumor suppressor egr-1 may be involved in the tumor progression in HCCR-1overexpressing cells. To further explain the tumorigenesis of $H C C R-1$, we determined the telomerase activity in PKC-activated HCCR-1-transfected cells. Consistent with a previous study [25],wild-type NIH/3T3 cells showed detectable telomerase activity (Figure $3 \mathrm{C}$ ). However, HCCR-1 gene transfection increased telomerase activity up to about 7 -fold when compared with wild-type cells. Reports show that PKC induces a marked increase in telomerase activity [26]. To determine whether the increased telomerase activity in $H C C R-1$ transfected cells is caused by $\mathrm{PKC}$, a kinase assay was performed. PKC activity of HCCR-1-transfected cells was increased by about 10-fold when compared with wild-type cells (Figure 3D)

\section{Discussion}

The conversion of normal cells into tumor cells involves changes in the activity of a number of distinct different genes and proteins in a cell. Although researchers have been able to transform normal mouse cells into tumorforming cells by introducing several cooperating oncogenes into these cells, human cells have been resistant to such transformation [8,27-29]. In this study, ectopic expression of $H C C R-1$ alone results in direct tumorigenic conversion of HEK-293 cells in vitro and in vivo.

Because NIH/3T3 cell is of mesenchymal origin [14],sarcoma would typically develop from oncogenetransformed NIH/3T3 [30]. But, nude mice bearing HCCR-1 allograft display characteristics of epithelial carcinomas. Because acquisition of epithelial properties by the fibroblast-derived cells mimics the mesenchymal to epithelial conversion of cells during the organogenesis of the kidney [20], we investigated whether HCCR-1 is expressed in the developing kidney. The developing nephrons in the cortex were not stained. But the basolateral plasma membranes of the developing collecting duct, which are derived from the ureteric bud [21],were especially reactive to HCCR-1 antibody. Because nephrogenesis is stimulated by a distinct ureteric signal, diffusionlimited basolateral molecules [22],which trigger mesenchymal to epithelial conversion, we propose that the HCCR-1 product may be a mesenchyme-derived regulatory factor [23] that stimulates morphogenesis of epithelia in the developmental process and mediates interactions between mesenchyme and epithelia during neoplastic transformation. Our study suggests that overexpression of HCCR-1 induces tumorigenesis, transdifferentiation and embryonic kidney development.

Transdifferentiation is a change from one differentiated phenotype to another involving morphological and functional phenotypic markers [31,32]. The conversion of a cell phenotype is likely to be accomplished by selective enhancement of gene expression, which controls the terminal developmental commitment of cells [33].There is little known about 'master switch' genes that determine a specific differentiation pathway and have the potential to induce the process in a cell originally destined for a differ- 
A
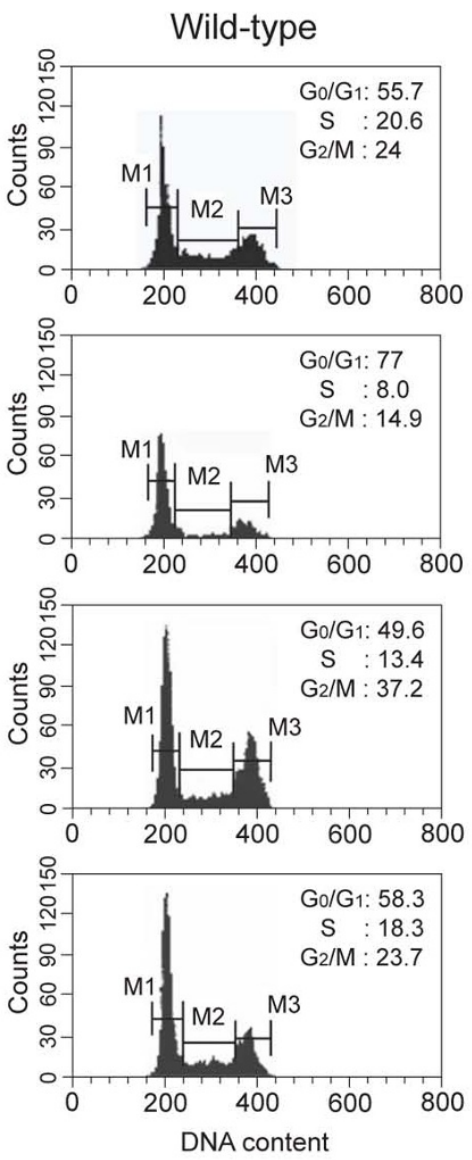

B

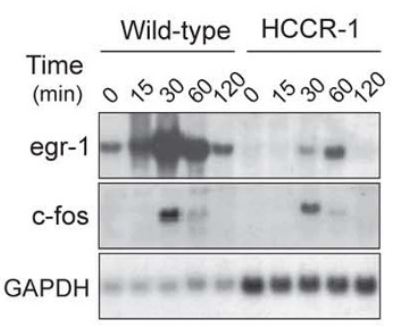

C
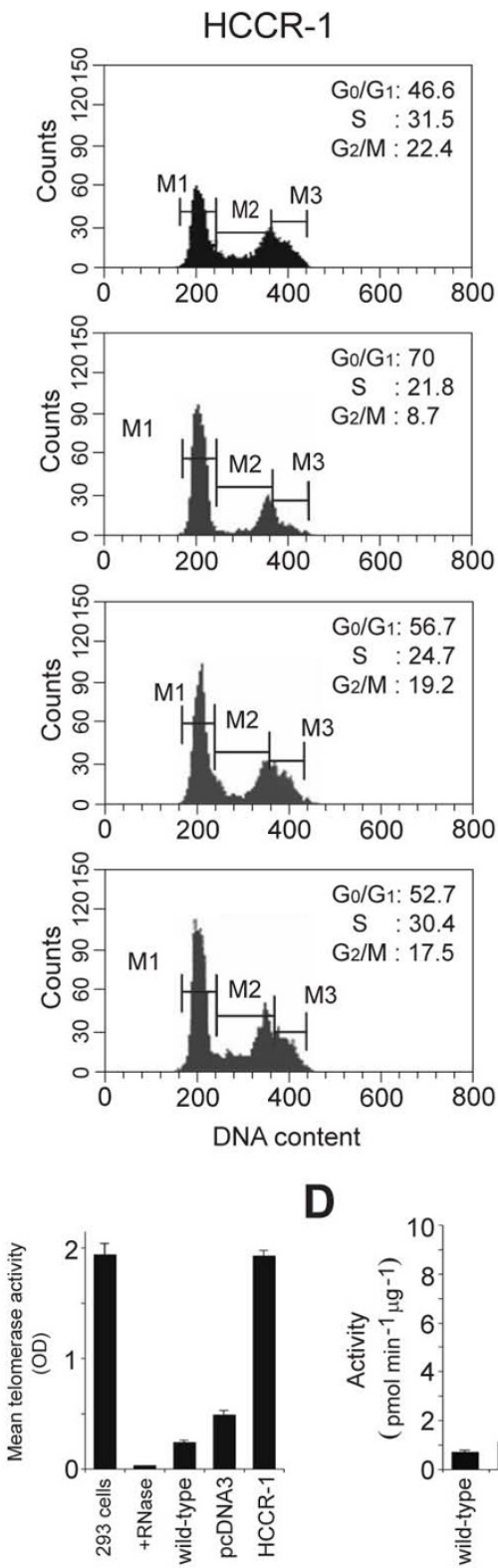

D

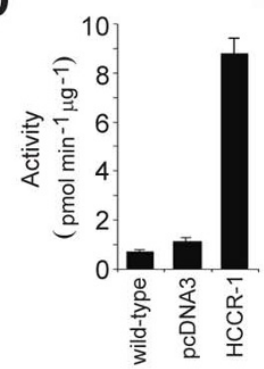

Figure 3 Molecular alterations in HCCR-1-induced tumorigenesis. A. Cell-cycle profiles of wild-type NIH/3T3 cell and HCCR-1-transfected cells from a separate experiment. Exponentially growing cells were trypsinized and DNA content was determined by flow cytometry. To assess the serumdependent cell cycle progression, cells were cultured in 0.5\% BCS for 36 houes. After incubation, cells were released with $20 \%$ serum and harvested. B. Tumor suppressor egr-1 expressions. HCCR-1-transfected cells were serum starved with 0.5\% BCS for 36 hours, and then stimulated with fresh $20 \%$ serum. Total RNA was isolated, transferred, and hybridized with 32P-labeled egr-1, c-fos, and GAPDH probe, respectively. C. Determination of telomerase activity. Human telomerase-positive embryonic kidney 293 cells, 293 cell extracts treated with RNase (+ RNase), wild-type NIH/3T3, cells transfected with vector alone and HCCR-1-transfected cells were analyzed by Telomerase PCR ELISA. Assays were performed according to the kit protocol with amounts of extracts equivalent to $1 \times 10^{3}$ cells. The telomerase activity in 293 cells, which served as a positive control, was abolished by pretreatment with RNase. Results are the average mean absorbance values from four separate experiments (means and 95\% confidence intervals) (wild-type versus HCCR-1, P< .05). D. Determination of PKC activity. Wild-type NIH/3T3, cells transfected with vector alone and HCCR-1-transfected cell extracts were prepared and assayed to determine PKC activity. Each value is the means and $95 \%$ confidence intervals of three independent experiments (wildtype versus HCCR-1, $P<0.05$ ). 
ent differentiation pathway [31].HCCR-1 might play multiple developmental roles by mediating a signal originating from the mesenchyme and received by epithelia. Mesenchymal signals are known to govern differentiation and morphogenesis of many epithelia, but the molecular nature of the signals is poorly understood. This expression pattern indicates that this mesenchymal factor can transmit morphogenetic signals in epithelia development and suggests a molecular mechanisim for mesenchymal epithelial interactions.

There is evidence to indicate that tumors develop secondarily to abnormalities in PKC-mediated signal transduction [34].Reports show that PKC induces a marked increase in telomerase activity [26].Besides tumor cells typically have acquired damage to genes that directly regulate their cell cycles [35]. Our study suggests that deregulation of HCCR-1 activity in mouse NIH/3T3 cells might result in the activation of PKC or telomerase, loss of particular cell cycle checkpoint controls, and downregulation of tumor suppressor egr-1, thereby predisposing $\mathrm{NIH} / 3 \mathrm{~T} 3$ cells to malignant conversion.

This present study suggests that $H C C R-1$ is an oncogene which induces the transformation of HEK293 and NIH3T3 cells. Likewise, our previous study also demonstrated that HCCR-1 is a mitochondrial out membrane protein and suppresses the apoptosis [36]. Consistent with this previous work, this study also reveals the antiapoptotic activity of $H C C R-1$ by reducing the expression of Egr-1, a direct regulator of multiple tumor suppressors including TGF beta1, PTEN, and p53. Therefore, both studies support that HCCR-1 is an oncogene either by suppressing apoptotic activities or by dysregulating Egr1, telomerase, or PKC activity. Since key functions related to apoptosis or anti-apoptosis often occur in mitochondria, it is not too surprising that HCCR-1 localizes to the mitochondria.

\section{Conclusions}

In conclusion, we converted normal cells into tumor cells by delivering HCCR-1 alone in combination with no other oncogenes. EMT and MET occurred in HCCR-1transfected tumor cells. In addition, $H C C R-1$ participates in induction of the c-kit proto-oncogene, in activation of PKC and telomerase activities, and cell cycle progression. While further studies are needed to characterize cellular functions and regulatory mechanisms, $H C C R-1$ protein is likely to be a candidate onco-developmental protein for cancer stem cell in the development of human cancer.

\section{Methods \\ Cell lines, construction of expression vector, and DNA transfection}

Human embryonic kidney (HEK) 293 (ATCC CRL-1573) and NIH/3T3 cells were obtained from the ATCC. HEK-
293/HCCR-1-V5 cell lineswere maintained in DMEM (Gibco) containing $200 \mu \mathrm{g} / \mathrm{ml} \mathrm{G418,10 \%} \mathrm{FBS} \mathrm{and} \mathrm{1 \%}$ PenStrep (Gibco).

Expression vector containing the coding region of HCCR-1 was constructed as follows. First, the SalI fragment was isolated from the prokaryotic expression vector, pCEV-LAC, which contains the entire HCCR-1 cDNA. Then, pcDNA3.1 (Invitrogen, CA) was digested with $X h o$ I to make a compatible end with SalI. A SalI fragment containing the $H C C R-1$ coding sequence was inserted into the XhoI-digested pcDNA3.1. Lipofectamine (Gibco BRL, Rockville, MD) was used to introduce the HCCR-1 expression vector into HEK-293 cells.

\section{Morphology and tumorigenecity}

Newly established cells grown in culture flasks were photographed by phase-contrast microscopy. To analyze tumorigenecity, $5 \times 10^{6}$ cells were injected subcutaneously into the posterior lateral aspect of the trunk of mice (5-week-old athymic nu/nu on BALB/c background). Nude mice were sacrificed when the subcutaneous tumors reached $1.5-2.5 \mathrm{~cm}$ in diameter.

\section{Colony-forming efficiency}

Five $\times 10^{3}$ viable cells were suspended in $1 \mathrm{ml}$ of $0.3 \%$ noble agar (Difco Laboratories Inc., Detroit, MI) made with complete media, and layered onto $0.6 \%$ agar in 35 $\mathrm{mm}$ plates. All samples were plated in quadruplicate. The number of cell colonies ( $>50$ cells/cluster) was estimated on days 21-28.

\section{Immunoblot analysis and immunohistochemistry}

For immunoblot analysis, cells were lysed in Laemmli sample buffer. Proteins were separated by $10 \%$ SDSPAGE and then electroblotted. The membranes were incubated with a rabbit polyclonal anti-HCCR-1 serum and proteins were revealed by an ECL-Western blot detection kit. We performed immunohistochemistry on cryosections $(5-\mu \mathrm{m})$ incubated with anti-vimentin, antikeratin, anti-EMA antibodies (DAKO), and polyclonal antibody raised against HCCR-1. Binding of primary antibody was visualized by biotinylated secondary antibody, avidin-horseradish peroxidase, and AEC as the chromogen

\section{PKC and telomerase activity assays}

PKC activity was measured using the SignaTECT ${ }^{\mathrm{TM}}$ Protein Kinase C Assay System (Promega, Madison, WI). PKC activity was defined as the difference in counts per minute incorporated into substrate in the absence and presence of phospholipid. Telomerase activity was determined by using the telomerase PCR-ELISA kit (Boehringer Mannheim). Immortalized human kidney cells (293 cells) provided in the kit were used as the positive 
control. A negative control was provided for human 293 cells by pretreatment with RNase.

\section{Cell cycle experiments}

Cells cultured at mid-log phase were growth arrested by incubation in medium containing $0.5 \%$ bovine calf serum for 36 hours. Cells to be analyzed for DNA content were harvested following trypsinization, and fixed in $70 \%$ ethanol. Fixed cells were then stained with propidium iodide. In brief, $50 \mu \mathrm{g} / \mathrm{ml}$ of propidium iodide staining solution (Sigma) and 100 units per $\mathrm{ml}$ of RNase A (Boerhinger Mannheim) were added to $2 \times 10^{6}$ cells. After incubation for 1 hour, cellular DNA content was determined by fluorescence analysis at $488 \mathrm{~nm}$ using a FACS Caliber (Becton Dickinson). A minimum of $1 \times 10^{4}$ cells per sample was analyzed with Modfit 5.2 software.

\section{Statistical analysis}

Data was analyzed by use of SAS software (SAS Institute, Cary, NC). One-way analysis of variance was used for comparing various outcome measures (e.g., PKC or telomerase levels) in different experimental conditions. The mean values and $95 \%$ confidence intervals for the outcome variables are shown in relevant figures. All reported $P$ values are two-sided and were considered to be statistically significant at the .05 level. $P$ values for comparing the difference between groups are adjusted by Dunnett's multiple comparisons.

\section{Additional material}

Additional file 1 Expression analysis of vimentin. (A, B) by immunofluorescence and epithelial markers such as $\mathrm{E}$-cadherin, $\mathrm{a}$-catenin, and $\beta$ catenin (C) by western blotting experiments. HEK-293 parental cells $(\mathbf{A})$ and HEK-293 stable clones for HCCR-1 (B) were stained for anti-vimentin antibodies. In C, HEK-293 parental cells (lanes 1 and 2), HEK-293 stable cells for HCCR-1 (lanes 3 and 4), and HEK-293 cells transfected with empty vectors (lanes 5 and 6 ) were analyzed by antibodies against E-cadherin, a-catenin, and $\beta$-catenin

Additional file $\mathbf{2}$ HCCR-1 gene in embryonic kidney development. A. Detection of HCCR-1 protein in fetal 16-, 18-, 20-, postnatal 1-, 7-, 14-day and adult rat kidney tissue extracts. Total proteins were subjected to SDS-PAGE. HCCR-1 positive bands were revealed by ECL-Western blot detection kit. $F$ and $P$ denote fetal and postnatal, respectively. B. Immunohistochemical staining of 20-day-old fetal rat kidney. Immunostaining was confined to the collecting ducts. Magnification, $\times 42$. C. Differential-interference contrast microscopy of 20-day-old fetal rat kidney illustrating HCCR-1 immunostaining in the basolateral plasma membrane of medullary collecting duct. Magnification, $\times 220$.

\section{Authors' contributions}

SH, HKK, JY, SK and SMS performed and designed experiments, analyzed data and assisted in writing the manuscript. YGP analyzed bioinformatics data, and YSL conducted histological analysis. YWK, TEK, YJC, SSJ, DWK, JK (from the Catholic) and SYS, YHL (from the Konkuk) were collaborators on the paper. JWK designed experiments, interpreted and assisted in writing the manuscript. SH and HKK contributed equally to this work. All authors read and approved the manuscript.

\section{Acknowledgements}

This research was supported by Basic Science Research Program through the National Research Foundation of Korea (NRF) funded by the Ministry of Education, Science and Technology (2009-0066496)

\section{Author Details}

'Molecular Genetic Laboratory, Catholic Medical Research Institute, The Catholic University of Korea, Seoul, Korea, 2Department of Clinical Pathology, College of Medicine, The Catholic University of Korea, Seoul, Korea, 3Department of Obstetrics and Gynecology, College of Medicine, The Catholic University of Korea, Seoul, Korea, ${ }^{4}$ Department of Microbiology, College of Medicine, The Catholic University of Korea, Seoul, Korea, ${ }^{5}$ Department of Neurosurgery, College of Medicine, The Catholic University of Korea, Seoul, Korea, ${ }^{\circ}$ Department of Internal Medicine, College of Medicine, The Catholic University of Korea, Seoul, Korea, ${ }^{7}$ Department of Biostatistics, College of Medicine, The Catholic University of Korea, Seoul, Korea, ${ }^{8}$ Department of Anatomy, College of Medicine, The Catholic University of Korea, Seoul, Korea and ${ }^{~}$ Department of Biomedical Science and Technology, Research Center for Transcription Control, Institute of Biomedical Science and Technology, Konkuk University, Seoul, Korea

Received: 21 October 2009 Accepted: 30 June 2010

Published: 30 June 2010

\section{References}

1. Eguchi G, Kodama R: Transdifferentiation. Curr Opin Cell Bio/ 1993, 5:1023-1028.

2. Strutz F, Muller GA, Neilson EG: Transdifferentiation. A new angle on renal fibrosis. Exp Nephrol 1996, 4:267-270.

3. Ko J, Lee YH, Hwang SY, Lee YS, Shin SM, Hwang JH, Kim J, Kim YW, Jang SW, Ryoo ZY, Kim IK, Namkoong SE, Kim JW: Identification and differential expression of novel human cervical cancer oncogene HCCR-2 in human cancers and its involvement in p53 stabilization. Oncogene 2003, 22:4679-4689.

4. Ko J, Shin SM, Oh YM, Lee YS, Ryoo ZY, Lee YH, Na DS, Kim JW: Transgenic mouse model for breast cancer: induction of breast cancer in novel oncogene HCCR-2 transgenic mice. Oncogene 2004, 23:1950-1953.

5. Yoon SK, Lim NK, Ha S-A, Park YG, Choi JY, Chung KW, Sun HS, Choi MJ, Chung J, Wands JR, Kim JW: The human cervical cancer oncogene protein is a biomarker for human hepatocellular carcinoma. Cancer Res 2004, 64:5434-5441.

6. Shin Seung Min, Chung Yeun Jun, Oh Seong Tack, Jeon Hae Myung, Hwang Lae Jeong, Namkoong Hong, Kim Hyun Kee, Cho Goang Won, Hur Soo Young, Kim Tae Eung, Lee Youn Soo, Park Yong Gyu, Ko Jesang, Kim Jin Woo: HCCR-1-interacting molecule "deleted in polyposis 1" plays a tumor-suppressor role in colon carcinogenesis. Gastroenterology 2006 130:2074-2086.

7. Land H, Parada LF, Weinberg RA: Tumorigenic conversion of primary embryo fibroblasts requires at least two cooperating oncogenes. Nature 1983, 304:596-602.

8. Hahn WC, Counter CM, Lundberg AS, Beijersbergen RL, Brooks MW, Weinberg RA: Creation of human tumour cells with defined genetic elements. Nature 1999, 400:464-468.

9. Dontu G, Al-Hajj M, Abdallah WM, Clarke MF, Wicha MS: Stem cells in normal breast development and breast cancer. Cell Prolif 2003, 36(Suppl. 1):59-72.

10. Lessard J, Sauvageau G: BMI-1 determines the proliferative capacity of normal and leukemic stem cells. Nature 2003, 423:255-260.

11. Pardal R, Clarke MF, Morrison SJ: Applying the principles of stem-cell biology to cancer. Nat Rev 2003, 3:895-902.

12. Reya T, Morrison SJ, Clarke MF, Weissman IL: Stem cells, cancer and cancer stem cells. Nature 2001, 414:105-111.

13. Spillane JB, Henderson MA: Cancer stem cells: a review. ANZ J Surg 2007. 77(6):464-468

14. Aaronson SA, Jainchill JL, Todaro GJ: Murine sarcoma virus transformation of BALB-3T3 cells: lack of dependence on murine leukemia virus. Proc Natl Acad Sci USA 1970, 66:1236-1243.

15. Yarden Y, Kuang WJ, Yang-Feng T, Coussens L, Munemitsu S, Dull TJ, Chen E, Schlessinger J, Francke U, Ullrich A: Human proto-oncogene c-Kit: a new cell surface receptor tyrosine kinase for an unidentified ligand. EMBO J 1987, 6:3341-3351 
16. Miettinen M, Lasota J: KIT (CD117): a review on expression in normal and neoplastic tissues, and mutations and their clinicopathologic correlation. Appl Immunohistochem Mol Morphol 2005, 13(3):205-220.

17. Karin M: Signal transduction form cell surface to nucleus in development and disease. FASEB J 1992, 6:2581-2590.

18. Yasuda A, Sawai H, Takahashi H, Ochi N, Matsuo Y, Funahashi H, Sato M, Okada Y, Takeyama H, Manabe T: Stem cell factor/c-kit receptor signaling enhances the proliferation and invasion of colorectal cancer cells through the PI3K/Akt pathway. Dig Dis Sci 2007, 52(9):2292-300.

19. Cho GW, Shin SM, Namkoong H, Kim HK, Ha SA, Hur SY, Kim TE, Chai YG, Kim JW: The phosphatidylinositol 3-kinase/Akt pathway regulates the HCCR-1 oncogene expression. Gene 2006, 384:18-26.

20. Tsarfaty I, Rong S, Resau JH, Rulong S, da Silva PP, Vande Woude GF: The Met proto-oncogene mesenchymal to epithelial cell conversion. Science 1994, 263:98-101.

21. Saxen L: Organogenesis of the Kidney. Cambridge: Cambridge University Press; 1987:88-128.

22. Barasch J, Pressler L, Connor J, Malik A: A ureteric bud cell line induces nephrogenesis in two steps by two distinct signals. Am J Physiol 1996 271:F50-61.

23. Barasch J, Yang J, Ware CB, Taga T, Yoshida K, Erdjument-Bromage H, Tempst P, Parravicini E, Malach S, Aranoff T, Oliver JA: Mesenchymal to epithelial conversion in rat metanephros is induced by LIF. Cell 1999, 99:377-386.

24. Huang RP, Darland T, Okamura D, Mercola D, Adamson ED: Suppression of v-sis-dependent transformation by the transcription factor, Egr-1. Oncogene 1994, 9:1367-1377.

25. Holt SE, Wright WE, Shay JW: Regulation of telomerase activity in immortal cell lines. Mol Cell Biol 1996, 16:2932-2939.

26. Li H, Zhao L, Yang Z, Funder JW, Liu JP: Telomerase is controlled by protein kinase $C$ alpha in human breast cancer cells. J Biol Chem 1998 273:33436-33442

27. Hjelle B, Liu E, Bishop JM: Oncogene v-src transforms and establishes embryonic rodent fibroblasts but not diploid human fibroblasts. Proc Natl Acad Sci USA 1988, 85:4355-4359.

28. Ron D, Tronick SR, Aaronson SA, Eva A: Molecular cloning and characterization of the human dbl proto-oncogene: evidence that its overexpression is sufficient to transform $\mathrm{NIH} / 3 \mathrm{~T} 3$ cells. EMBO J 1988, 7:2465-2473.

29. May WA, Arvand A, Thompson AD, Braun BS, Wright M, Denny CT: EWS/ FLI1-induced manic fringe renders NIH 3T3 cells tumorigenic. Nat Genet 1997, 17:495-497.

30. Lieberman MW, Lebovitz RM: Neoplasia. In Anderson's Pathology Edited by: Damjanov I, Linder J. St. Louis, MO: Mosby; 1996:518.

31. Boukamp P: Transdifferentiation induced by gene transfer. Semin Cell Biol 1995, 6:157-163.

32. Yuan $S$, Rosenberg L, Paraskevas S, Agapitos D, Duguid WP: Transdifferentiation of human islets to pancreatic ductal cells in collagen matrix culture. Differentiation 1996, 61:67-75.

33. Beresford WA: Direct transdifferentiation: can cells change their phenotype without dividing? Cell Differ 1990, 29:81-93.

34. Benzil DL, Finkelstein SD, Epstein MH, Finch PW: Expression pattern of alpha-protein kinase $\mathrm{C}$ in human astrocytomas indicates a role in malignant progression. Cancer Res 1992, 52:2951-2956.

35. Sherr CJ: Cancer cell cycles. Science 1996, 274:1672-1677.

36. Cho Goang-Won, Shin Seung Min, Kim Hyun Kee, Ha Seon-Ah, Kim Sanghee, Yoon Joo-Hee, Hur Soo Young, Kim Tae Eung, Kim Jin Woo: HCCR-1, a novel oncogene, encodes a mitochondrial outer membrane protein and suppresses the UVC-induced apoptosis. BMC Cell Biology 2007, 8:1-12.

doi: $10.1186 / 1471-2121-11-49$

Cite this article as: $\mathrm{Ha}$ et al., Transdifferentiation-inducing HCCR-1 oncogene BMC Cell Biology 2010, 11:49

\section{Submit your next manuscript to BioMed Central} and take full advantage of:

- Convenient online submission

- Thorough peer review

- No space constraints or color figure charges

- Immediate publication on acceptance

- Inclusion in PubMed, CAS, Scopus and Google Scholar

- Research which is freely available for redistribution

Submit your manuscript at www.biomedcentral.com/submit
C Biomed Central 